OPEN ACCESS

Edited by:

Thierry Moulin,

Centre Hospitalier Universitaire de

Besançon, France

Reviewed by:

Francesco Corea,

Azienda USL Umbria 2, Italy Elisabeth Medeiros De Bustos,

CHU Jean Minjoz, France

${ }^{*}$ Correspondence: Peter Müller-Barna

peter.mueller-barna@ muenchen-klinik.de

Specialty section:

This article was submitted to

Stroke,

a section of the journal

Frontiers in Neurology

Received: 29 August 2021 Accepted: 31 January 2022 Published: 02 March 2022

Citation:

Müller-Barna P, Leinweber C, Pfaffenrath J, Schütt-Becker N, von

Martial R, Greck S, Hubert N, Rambold H, Haberl $R$ and Hubert GJ (2022) Identification of Stroke and TIA

in Patients With Acute Dizziness,

Vertigo or Imbalance in Emergency

Departments of Primary Care Hospitals: Early Experiences With a

Network-Based Telemedical

Approach. Front. Neurol. 13:766685.

doi: 10.3389/fneur.2022.766685

\section{Identification of Stroke and TIA in Patients With Acute Dizziness, Vertigo or Imbalance in Emergency Departments of Primary Care Hospitals: Early Experiences With a Network-Based Telemedical Approach}

\author{
Peter Müller-Barna ${ }^{1 *}$, Christina Leinweber ${ }^{1}$, Julia Pfaffenrath ${ }^{1}$, Nina Schütt-Becker ${ }^{1}$, \\ Rascha von Martial ${ }^{1}$, Susanne Greck ${ }^{1}$, Nikolai Hubert ${ }^{1}$, Holger Rambold ${ }^{2,3,4}$, \\ Roman Haberl ${ }^{1}$ and Gordian Jan Hubert ${ }^{1}$ \\ ${ }^{1}$ Department of Neurology, TEMPiS Telestroke Center, München Klinik, Academic Teaching Hospital of the \\ Ludwig-Maximilians-University, Munich, Germany, ${ }^{2}$ Department of Neurology, InnKlinikum Altötting, Altötting, Germany, \\ ${ }^{3}$ Department of Neurology, University of Regensburg, Regensburg, Germany, ${ }^{4}$ MVZ Kliniken Mühldorf, Mühldorf am Inn, \\ Germany
}

Background: Acute dizziness, vertigo, and imbalance are frequent and difficult to interpret symptoms in the emergency department (ED). Primary care hospitals often lack the expertise to identify stroke or TIA as underlying causes. A telemedical approach based on telestroke networks may offer adequate diagnostics and treatment.

Aim: The aim of this study is to evaluate the accuracy of a novel ED algorithm in differentiating between peripheral and central vestibular causes.

Methods: Within the Telemedical Project for Integrative Stroke Care (TEMPiS), a telemedical application including a videooculography (VOG) system was introduced in 2018 in 19 primary care spoke hospitals. An ED triage algorithm was established for all patients with acute dizziness, vertigo, or imbalance of unknown cause (ADVIUC) as a leading complaint. In three predefined months, all ADVIUC cases were prospectively registered and discharge letters analyzed. Accuracy of the ED triage algorithm in differentiation between central and peripheral vestibular cases was analyzed by comparison of ED diagnoses to final discharge diagnoses. The rate of missed strokes was calculated in relation to all cases with a suitable brain imaging. Acceptance of teleconsultants and physicians in spoke hospitals was assessed by surveys.

Results: A total number of 388 ADVIUC cases were collected, with a median of 12 cases per months and hospital (IQR 8-14.5). The most frequent hospital discharge diagnoses are vestibular neuritis (22\%), stroke/TIA (18\%), benign paroxysmal positioning vertigo (18\%), and dizziness due to internal medicine causes (15\%). Detection of a central vestibular cause by the ED triage algorithm has a high sensitivity (98.6\%), albeit poor 
specificity (45.9\%). One stroke out of 32 verified by brain scan was missed (3.1\%). User satisfaction, helpfulness of the project, improvement of care, personal competence, and satisfaction about handling of the VOG systems were rated consistently positive.

Discussion: The concept shows good acceptance for a telemedical and network-based approach to manage ADVIUC cases in the ED of primary care hospitals. Identification of stroke cases is accurate, while specificity needs further improvement. The concept could be a major step toward a broadly available state of the art diagnostics and therapy for patients with ADVIUC in primary care hospitals.

Keywords: dizziness, vertigo, telemedicine, emergency medicine, stroke, diagnostic method, acute vestibular syndrome

\section{INTRODUCTION}

Acute dizziness and vertigo are frequent and difficult to diagnose symptoms in the emergency department (ED) (1-3). Since stroke is the underlying cause in about $5 \%$ of these cases $(4,5)$ but missed in about $35 \%$ of stroke cases, especially when symptoms are mild and transient (6-9), a specific concept is required. Therefore, a workflow in the ED is desirable which identifies all stroke cases correctly for rapid admission to a stroke unit and applies immediate therapy such as intravenous thrombolysis if indicated. The most promising approach seems to use a battery of different oculomotor tests like the HINTS (head impulse nystagmus - test of skew) or the HINTS plus exam (10-14), which identify strokes based on clinical examinations better than an initial MRI $(9,12)$. Adding the video head impulse test (vHIT) to quantify the vestibular ocular reflex further improves the effectiveness of the algorithm (15-17).

As those tests are applied by trained neurootologists, our challenge is to bring the necessary expertise to the bedside in ED of primary care hospitals, which mostly do not employ a neurologist in the ED. Our favored solution is telemedicine, as is already used with success in treating stroke, known as telestroke $(18,19)$. A telemedical consultation program for acutely dizzy patients bringing neurootological expertise in the ED of the same hospital reported higher diagnostic accuracy and lower employment of computed tomography scans (20). The employment of smartphones for vHIT measurement may facilitate this development in the future (21). A clinical trial comparing video-oculography (VOG) guided care to standard care in EDs is ongoing (Acute Video-Oculography for Vertigo in Emergency Rooms for Rapid Triage [AVERT] trial) (22).

Current telestroke networks seem ideal for the additional implementation of telemedical care for acutely dizzy patients, as there are close synergies to be expected (medically - because of the underlying etiology of stroke - and technically - because of the similar infrastructure needed). The logistics developed

\footnotetext{
Abbreviations: ADVIUC, acute dizziness, vertigo, or imbalance of unknown cause; BPPV, benign paroxysmal positional vertigo; HINTS, head impulse nystagmus - test of skew; IQR, interquartile range; TEMPiS, Telemedical Project for Integrative Stroke Care; TiTrATE, timing, triggers, and targeted bedside eye examinations; TIA, transient ischemic attack; vHIT, video head impulse test; VOG, videooculography.
}

for telestroke could be extended for dizzy patients, regarding teleconsultation workflow, specific treatment standards, training, and quality management. Additional resources needed are mainly one expert neurootologist and one vestibular rehabilitation therapist in the network center, VOG systems in all spokes and sufficient resources for training and quality management (23).

This study evaluates our telematic approach to diagnose acute dizziness, vertigo, and imbalance in our telemedical stroke network TEMPiS in Bavaria, Germany, with the main focus on the diagnostic accuracy of the ED algorithm in differentiating between peripheral and central vestibular diseases. Results of the remote VOG examination including telemedical HINTS and vHIT are reported in another paper (24).

\section{MATERIALS AND METHODS}

As telestroke is already implemented in our region of SouthEast Bavaria, we built our concept on this telematic system. The Telemedical Project for Integrative Stroke Care (TEMPiS) is a stroke network consisting of two hubs and 24 spokes and was implemented in $2003(18,19)$. A total of 7,427 and 7,337 teleconsultations were performed in 2019 and 2020, respectively. TeleVertigo was introduced in 2018 in 19 spoke hospitals as an add-on to the preexisting telestroke collaboration (Figure 1). The detailed concept was already published (23). The catchment area of the 19 hospitals sums up to a population of $1,987,414$ inhabitants (25). Six of these spokes have a neurology department on-site with $24 / 7$ availability of a neurologist.

In brief, our innovative telemedical concept addresses all patients who were admitted to an ED of the participating primary care hospitals due to acute dizziness, vertigo, or imbalance of unknown cause (ADVIUC) as a leading complaint (Figure 2). The key technical diagnostic tool is a VOG system embedded in a standard videoconferencing system. In contrast to standard videoconferencing systems (26), the added VOG system enables vestibular testing by telemedicine. The concept comprises three stages: (1) telemedical triage in the ED (including vHIT and fast track VOG; Figure 3) for reliable and rapid decision-making on acute treatment, including stroke treatment if indicated: This triage uses a telemedical examination of oculomotor signs, the vHIT and the diagnostic Dix-Hallpike-maneuvers for 


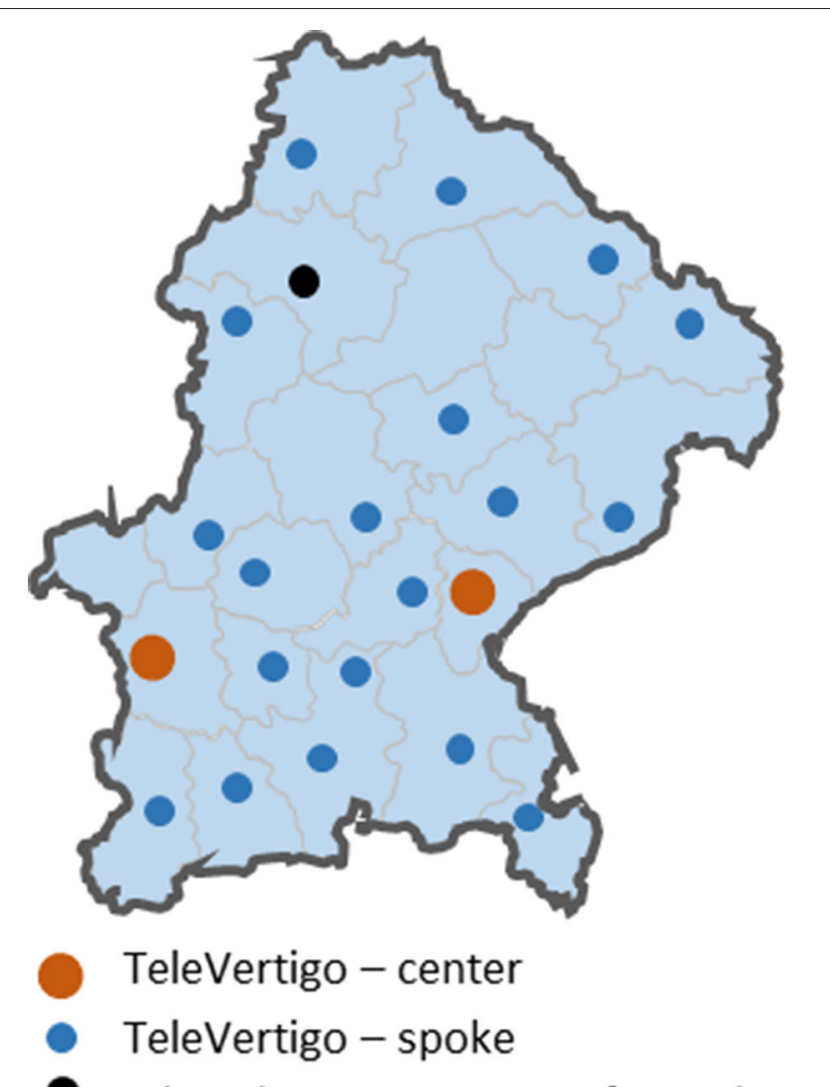

TeleStroke center: University of Regensburg

FIGURE 1 | Map of the southeastern part of Bavaria, Germany with all TeleVertigo hospitals.

benign paroxysmal positional vertigo (BPPV) of the posterior semicircular canals. For this purpose, a VOG system ("video goggles"; ICS Impulse Type 1085 in combination with the OTOsuite Vestibular software, version 4.10 Build 1341, Natus Medical Denmark ApS, Taastrup, Denmark) was included in the videoconferencing system in all participating hospitals; (2) elective clinical examination including quantitative VOG and finding of the causal diagnosis: In cases of ADVIUC, an elective neurological examination by the vascular neurologist is done next day. Vascular neurologists are thoroughly trained within this project in diagnosing dizziness and vertigo. Additionally, a VOG including nystagmus detection, tests for skew deviation, visually guided saccades, smooth pursuit eye movements, vHIT, and Dix-Hallpike-maneuvers is performed by trained technicians or physical therapists. On demand, a neurootologist of the network center evaluates this VOG remotely and supports spoke physicians in diagnosis and therapy. (3) Adequate treatment including vestibular rehabilitation and canalith repositioning procedures: Physiotherapy was established for all patients with ongoing dizziness, vertigo, or imbalance, including canalith repositioning maneuvers and vestibular rehabilitation. To obtain high standards, a physical therapist of the network center specialized in vestibular disorders offers regular training to the physical therapists in the spoke hospitals.

In 2018, we developed project standards for acute and elective diagnosis and treatment of patients with dizziness, vertigo, and imbalance, and also specific diagnostic and treatment standards for benign paroxysmal positional vertigo, vestibular neuritis, and dizziness of central origin, based on the criteria of the Bárány Society (27-29). We trained 180 physicians, therapists, and technicians of the participating hospitals in these standards in 15 sessions lasting $6 \mathrm{~h}$ each, consisting of $3.5 \mathrm{~h}$ of theoretical training and $2.5 \mathrm{~h}$ of practical training. An exit test or any other credential was not collected.

\section{Study Protocol}

For project evaluation, evaluation periods with full documentation of all admitted patients in the EDs of the participating hospitals were predefined. In compliance with the capacity of participating primary care hospitals, we tailored the evaluation effort to 3 months (11/2019,3/2020, and 10/2020). A screening registry was designed, and all participating hospitals were asked to register all patients with the leading symptom of acute dizziness, vertigo, or imbalance of unknown cause (ADVIUC) in screening lists. "Unknown cause" was defined as follows: (1) In the ED, no history of episodic vertigo or dizziness with the same quality of symptoms like in the actual presentation was reported, and (2) in the first short triage done by ED physicians, an underlying reason for the acute dizziness symptom is not obvious. This triage includes a short internal medicine and neurological examination and also the assessment of vital parameters (heart rate, blood pressure, temperature, oxygen saturation), an ECG and basic blood parameters (small blood count, natrium, potassium and, if necessary, inflammation parameters, and D-dimer). Therefore, patients with an obvious hemiparesis or dyspnea were excluded, whereas patients with a subtle focal neurological deficit or mild internistic symptoms were included. In the ADVIUC analysis, all cases with symptoms lasting more than $72 \mathrm{~h}$ were excluded.

For all ADVIUC cases, discharge letters were requested, and relevant information was extracted by neurootologists in the stroke center (CL, PMB). Base data collected are as follows: age, sex, relevant risk factors, relevant symptoms, and hospital discharge diagnoses.

Accuracy of the triage algorithm was focused on cases with vestibular disorders. We compared the ED diagnoses to final discharge diagnoses for all central and peripheral vestibular cases according to the final discharge diagnosis. ED diagnoses were categorized as peripheral if diagnostic criteria for vestibular neuritis or BPPV were fulfilled. Cases with central signs (e.g., a direction-changing nystagmus or a skew deviation) or constellations suggestive for a central lesion (e.g., direction-fixed horizontal nystagmus, but physiological vHIT) or unclear constellations (e.g., no peripheral and central vestibular symptoms) were categorized as central.

In most ADVIUC cases without clear evidence for peripheral or internal medicine cause, a CT scan was performed in the ED. In all ADVIUC cases suspicious for a central etiology, cranial MRI was recommended between days 3 and 5 . The written results 


\section{TeleVertigo}

\begin{tabular}{|c|c|c|c|c|c|}
\hline Aim & \multicolumn{5}{|c|}{ Accurate identification of stroke/TIA cases \& improvement of in-hospital quality of care } \\
\hline Population & \multicolumn{5}{|c|}{ Acute dizziness, vertigo or imbalance of unknown cause (ADVIUC) in the ED of primary care hospitals } \\
\hline $\begin{array}{l}\text { Project } \\
\text { steps }\end{array}$ & $\begin{array}{l}\text { Treatment } \\
\text { Standards } \\
\text { - ED triage incl. acute } \\
\text { VOG } \\
\text { - Elective examination } \\
\text { incl. elective VOG } \\
\text { - Treatment incl. } \\
\text { specialized } \\
\text { physiotherapy } \\
\text { Focus on: } \\
\text { - ADVIUC from central } \\
\text { cause (e.g. stroke) } \\
\text { - vestibular neuritis } \\
\text { - BPPV } \\
\text { - internal medicine } \\
\text { causes for ADVIUC }\end{array}$ & $\begin{array}{l}\text { Teaching \& } \\
\text { Trainings } \\
\text { for: } \\
\text { - teleconsultants } \\
\text { - ED physicians } \\
\text { - ED nurses } \\
\text { - on-site } \\
\text { neurologists } \\
\text { - on-site } \\
\text { technicians } \\
\text { - on-site } \\
\text { physiotherapists } \\
\\
\text { done by: } \\
\text { - neuro-otologist } \\
\text { (center) } \\
\text { - vestibular } \\
\text { therapist(center) }\end{array}$ & $\begin{array}{l}\text { Stage 1: ED tria } \\
\text { - } 24 / 7 \text { available } \\
\text { - ED physician ano } \\
\text { - internistic and n } \\
\text { otological exami } \\
\text { diagnostic mane } \\
\text { - Aim: rapid triage } \\
\text { Stage 2: Electi } \\
\text { - next working da } \\
\text { - on-site neurolog } \\
\text { - elective VOG on } \\
\text { - consultation of } n \\
\text { demand } \\
\text { Stage } 3: \text { Treatr } \\
\text { - early during hos } \\
\text { - vestibular rehab } \\
\text { procedures by tr } \\
\text { consultation of s } \\
\text { the center on de }\end{array}$ & $\begin{array}{l}\text { sultant } \\
\text { al examination, neuro- } \\
\text { VOG) incl. HINTS and vHIT, } \\
\text { BPPV } \\
\text { ve unit or normal ward } \\
\text { mination } \\
\text { ologist in the center on } \\
\\
\text { and canalith repositioning } \\
\text { hysiotherapists } \\
\text { edvestibular therapist in }\end{array}$ & $\begin{array}{l}\text { Quality } \\
\text { Management } \\
\text { - quality circles } \\
\text { - quality monitoring } \\
\text { - evolution of } \\
\text { system of care }\end{array}$ \\
\hline $\begin{array}{l}\text { Techno- } \\
\text { logy }\end{array}$ & \multicolumn{3}{|c|}{ VOG systems in each spoke } & \multicolumn{2}{|c|}{$\begin{array}{l}\text { Display of an additional video of one eye } \\
\text { in the center }\end{array}$} \\
\hline Basis & \multicolumn{3}{|c|}{ Telestroke network with (tele-)stroke units in each spoke } & \multicolumn{2}{|c|}{$\begin{array}{l}\text { Network center with expertise in } \\
\text { neuro-otology \& vestibular rehabiltation }\end{array}$} \\
\hline
\end{tabular}

FIGURE 2 | Overview of the major components of the TeleVertigo project.

of all brain scans performed were collected and evaluated by experienced vascular neurologists centrally (CL, PMB). For the evaluation of missed strokes, all ADVIUC cases with an adequate brain imaging were considered. All MRI scans were considered as adequate, whereas CT scans were counted only if pathologic. All cases in the spoke hospitals with CT or MRI evidence of stroke, which were not primarily admitted to stroke units, were counted as missed strokes.

For all acute telemedical ED triage examinations, subjective satisfaction and added value were documented by the teleconsultants. Accordingly, the same items were documented for all elective VOGs by the physicians who made the finding. For further project evaluation, an online questionnaire was sent to all participating hospitals at the end of the project phase in November 2020. We wrote to our medical contact persons and also to therapists and technicians with the request to forward the questionnaire to their colleagues. The questionnaire asked for the subjective assessment of how helpful measures within the project were perceived, whether the care of patients with dizziness in their own hospital was improved, whether their own competence could be expanded, how satisfactory the implementation of the VOG was perceived, and whether a continuation of the application was desired. Answers were given anonymously.

\section{Outcomes and Statistics}

Primary outcome of this evaluation is the accuracy of the ED triage algorithm in differentiation between peripheral and central vestibular disease. Secondary outcomes are rate of missed strokes in acutely dizzy patients, improvement of diagnostic quality, and acceptance of physicians in spoke hospitals.

We applied descriptive statistics. Due to non-normal distribution of cases, data are presented as median and interquartile range. All patient data are derived from our anonymized and project-related quality register. According to German legislation, no patient consent is required for documentation in routine observational quality registries. 


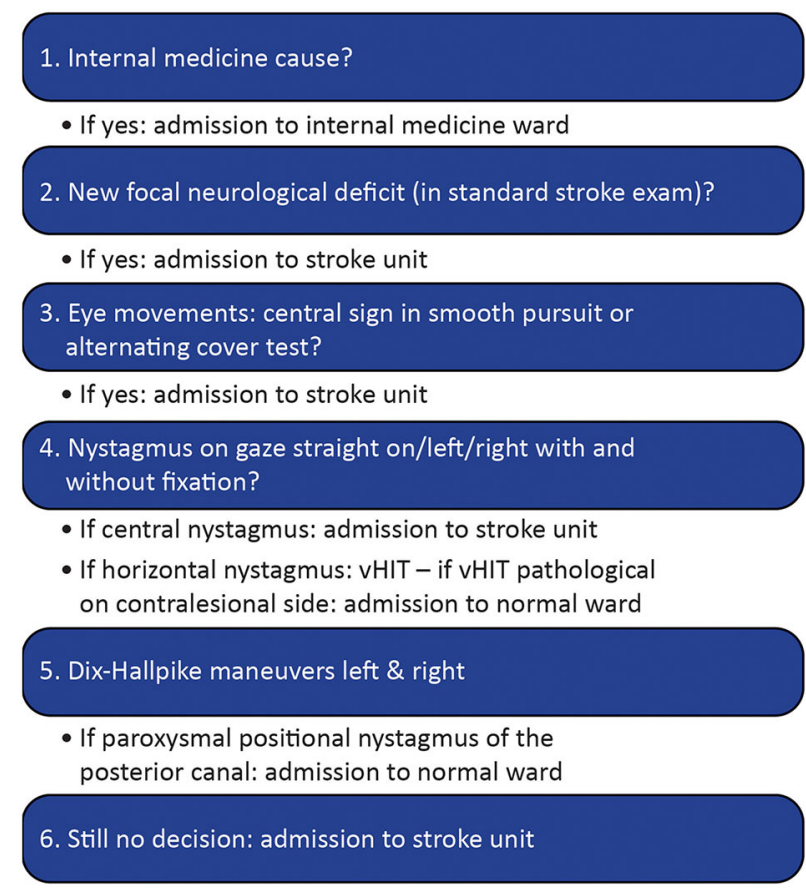

FIGURE 3 | ED triage algorithm for all cases of acute dizziness, vertigo, or imbalance.

\section{RESULTS}

Screening lists were expected from all 19 hospitals in all of the three evaluation months (total $n=57$ ) and provided from 18 hospitals in 40 evaluation months $(40 / 57=70 \%)$. We had to exclude 8 lists due to implausible data, with 32 remaining lists from 15 hospitals $(32 / 57=56 \%)$ and a total of 469 cases with the leading symptom of dizziness, vertigo, or imbalance in the ED. Response rate improved gradually over time from 6 to 12 to 14 hospitals with valid screening lists. The median number of cases was 13.5 per month and hospital [interquartile range (IQR) 10-21]. After exclusion of 81 cases with symptoms lasting more than $72 \mathrm{~h}$, a total number of 388 ADVIUC cases remained with a median number of 12 per month and hospital (IQR 8-14.5; see Table 1).

Baseline characteristics, risk factors, and symptoms of the 388 acute cases are given in Table 2. In 11.9\% of cases, there is a history of dizziness or vertigo, but of different symptom quality. In most cases (87.9\%), onset was reported as hyperacute. Nystagmus was detected in $51.0 \%$ of cases.

Distribution of hospital discharge diagnoses of these cases is displayed in Table 3 with vestibular neuritis, stroke/TIA and BPPV (each about 20\%), and dizziness of internal medicine causes (15\%) as the most frequent diagnoses.

Accuracy of the ED triage algorithm was calculated in vestibular cases compared to the final discharge diagnosis (see Figure 4). Sensitivity for detecting a central lesion was $73 / 74$ (98.6\%) and specificity $83 / 181$ (45.9\%). The positive predictive value is $73 / 171(42.7 \%)$ and the negative predictive value $83 / 84$ (98.8\%) (see Table 4).
TABLE 1 | Number of cases per hospital and evaluation month with acute $(<72 \mathrm{~h}$ from onset) dizziness, vertigo, or imbalance of unknown cause (ADVIUC) as a leading symptom for ED admission.

\begin{tabular}{|c|c|c|c|c|}
\hline & Month 1 & Month 2 & Month 3 & Cases per year \\
\hline Agatharied & 10 & 12 & 14 & 144 \\
\hline Bad Reichenhall & 9 & 8 & 7 & 96 \\
\hline Bad Tölz & 7 & 11 & 13 & 124 \\
\hline Burglengenfeld & & 8 & 12 & 120 \\
\hline Cham & & 12 & & 144 \\
\hline Ebersberg & & 10 & 5 & 90 \\
\hline Eggenfelden & & & 25 & 300 \\
\hline Erding & & & 12 & 144 \\
\hline Freising & & 12 & 16 & 168 \\
\hline Mühldorf & & 7 & 12 & 114 \\
\hline Rosenheim & 16 & 17 & 17 & 200 \\
\hline Rotthalmünster & & 12 & 6 & 108 \\
\hline Traunstein & 18 & 20 & 24 & 248 \\
\hline Vilsbiburg & & & 8 & 96 \\
\hline Wasserburg & 13 & 9 & 6 & 112 \\
\hline Total & 73 & 138 & 177 & 2,208 \\
\hline Median (IQR) & & $12(8-14.5)$ & & $124(110-184)$ \\
\hline
\end{tabular}

Annual number of cases are extrapolated from monthly numbers.

In 196 cases, an adequate brain imaging (MRI in 192 cases and CT in 4 cases) was performed with a brain lesion matching the symptoms in 36 cases $(36 / 196=18.4 \%)$. In four cases, lesions were not due to stroke: 1 acoustic neurinoma, 1 inflammatory lesion, 1 cerebral tumor, and 1 PICA aneurysm with compression of the brainstem. Three out of 32 stroke lesions were detected in CT scans (two ischemic cerebellar and one ischemic brainstem lesion), the remaining by MRI scans. A number of 16 out of 32 strokes were located predominantly in the cerebellum, 11/32 strokes in the brainstem, and 5/32 strokes in the cortex. One stroke was a cerebellar hemorrhage, the remaining ischemic. With the ED triage algorithm, $31 / 32$ (96.9\%) strokes confirmed by imaging were detected correctly in the ED. One stroke was missed $(3.1 \%)$.

User satisfaction, the subjective sensation of safety in the clinical examination and diagnostic interpretation and the subjective added value in the concrete case by application of the acute and elective VOG are generally high, as stated by the teleconsultants and on-site physicians (see Figure 5). A total of 32 colleagues responded to our anonymous online questionnaire in November 2020. The total of 59\% of them are physicians, $34 \%$ therapists and 6\% technicians. Questions about helpfulness of the project, improvement of care on ADVIUC patients, and personal competence and also satisfaction about handling of the elective VOGs were rated consistently positive (for details see Figure 6). Slightly lower rates ( $80 \%$ positive) were recorded concerning helpfulness and satisfaction with the handling of acute VOGs. All responders voted for a continuation of the project. 
TABLE 2 | Baseline characteristics of all cases with an acute leading symptom of dizziness, vertigo, or imbalance of unknown cause (ADVIUC).

\begin{tabular}{|c|c|c|c|}
\hline & $n$ & $N$ & $\%$ \\
\hline Age [median; IQR (years)] & $65(54-78)$ & & \\
\hline Female sex & 210 & 388 & $54.1 \%$ \\
\hline \multicolumn{4}{|l|}{ Risk factors } \\
\hline Arterial hypertension & 224 & 388 & $57.7 \%$ \\
\hline Diabetes mellitus & 68 & 388 & $17.5 \%$ \\
\hline Hypercholesterinemia & 146 & 388 & $37.6 \%$ \\
\hline (ex-)smoker & 59 & 388 & $15.2 \%$ \\
\hline Atrial fibrilation & 46 & 376 & $12.2 \%$ \\
\hline Osteoporosis & 17 & 359 & $4.7 \%$ \\
\hline History of dizziness/vertigo & 43 & 360 & $11.9 \%$ \\
\hline \multicolumn{4}{|l|}{ Symptoms } \\
\hline Hyperacute onset & 340 & 387 & $87.9 \%$ \\
\hline Dizziness or vertigo & 386 & 388 & $99.5 \%$ \\
\hline Vertigo only & 206 & 370 & $55.7 \%$ \\
\hline Imbalance & 235 & 359 & $65.5 \%$ \\
\hline Nystagmus & 197 & 386 & $51.0 \%$ \\
\hline Nausea & 246 & 343 & $71.7 \%$ \\
\hline Vomiting & 153 & 340 & $45.0 \%$ \\
\hline New neck pain or headache & 49 & 263 & $18.6 \%$ \\
\hline Known headache & 22 & 388 & $5.7 \%$ \\
\hline New tinnitus & 21 & 214 & $9.8 \%$ \\
\hline New hearing disturbance & 14 & 211 & $6.6 \%$ \\
\hline New ear symptoms (others) & 20 & 211 & $9.5 \%$ \\
\hline New phono- or photophobia & 8 & 163 & $4.9 \%$ \\
\hline
\end{tabular}

Missing cases are due to missing information in available hospital discharge letters.

TABLE 3 | Distribution of hospital discharge diagnoses of all cases $(n=388)$ with an acute leading symptom of dizziness, vertigo, or imbalance of unknown cause (ADVIUC).

\begin{tabular}{lcc}
\hline Diagnosis & $\boldsymbol{n}$ & $\%$ \\
\hline Vestibular neuritis & 87 & $22.4 \%$ \\
Vertebrobasilar stroke or TIA & 68 & $17.5 \%$ \\
BPPV & 68 & $17.5 \%$ \\
Dizziness of internal medicine cause & 57 & $14.7 \%$ \\
Meniere's disease & 26 & $6.7 \%$ \\
Functional disorder & 7 & $1.8 \%$ \\
Vestibular migraine & 7 & $1.8 \%$ \\
Inflammatory CNS disease & 6 & $1.5 \%$ \\
Others & 3 & $0.8 \%$ \\
Unclear & 59 & $15.2 \%$
\end{tabular}

"Others include one case of bilateral vestibulopathy, vestibular paroxysm, and polyneuropathy each

\section{DISCUSSION}

We report on our experience of a telemedical approach to improve quality of care in patients with acute dizziness, vertigo, or imbalance in primary care hospitals. The approach is based on telestroke networks and comprises multiple components including the implementation of standards, training of ED physicians, on-site neurologists, on-site therapists and teleconsultants, telemedical examinations, and implementation of VOG including vHIT (see Figure 2). The implementation is challenging and needs relevant resources and time, but our data demonstrate its feasibility. The approach distinguishes central (mostly stroke) from peripheral vestibular disorders with high sensitivity, albeit poor specificity. The vast majority of colleagues involved report subjective satisfaction and added diagnostic value and also the desire to continue the project.

The median monthly number of ADVIUC cases in the EDs of our primary care hospitals is 12 (IQR 8 - 14.5). Our data allow us to estimate an incidence, because catchment areas of our primary care spoke hospitals correspond largely to the correlating county, as we know from earlier evaluations of stroke cases (30). An extrapolated annual number of 2,208 ADVIUC cases correspond to a population of $1,987,414$ inhabitants, resulting in an annual incidence of 111 cases per 100,000 inhabitants. This is in line with population-based studies performed by retrospective medical record reviews. A study from Sweden (4) reports an incidence of acute vestibular syndrome in the ED of 92 per 100,000 inhabitants per year, and another from Texas (6), reports 1,297 isolated dizziness symptoms as the principal presenting complaint in a 3.5 -year period in proportion to about 300,000 inhabitants, and therefore resulting in an incidence of 123.5 cases per 100,000 inhabitants per year.

Our ADVIUC cases in ED of primary care hospitals resulted in the final diagnosis of vestibular neuritis, stroke/TIA and BPPV in about $20 \%$ of cases each, and dizziness of internal medicine cause in about $15 \%$. These figures differ relevantly from other publications. Ljungren report $10 \%$ stroke/TIA in their sample of acute vertigo in the ED (4). However, it must be mentioned that only $45 \%$ of cases got a medical diagnose, whereas $55 \%$ were coded with a symptom diagnose. In an US sample of dizziness presentations in the $\mathrm{ED}, 4 \%$ resulted in a diagnosis of stroke/TIA, $33 \%$ otologic/vestibular, $21 \%$ cardiovascular, and $11 \%$ respiratory and metabolic (5). Noteworthy is again that only $49 \%$ of cases got a medical diagnose and the sample was not restricted to acute cases without an obvious underlying reason for the chief complaint of dizziness. Therefore, we believe that differences are explained by a selection bias due to different sampling definitions and an additional coding bias (in our sample in $15 \%$ of cases the final diagnosis remained unclear). Data for acute vertigo/dizziness from the ED of a tertiary referral center seem to match our data quite wellconsidering that recurrent manifestations of episodic syndromes are not excluded and medical disorders may be included more frequently (31).

The high proportion of $20 \%$ of stroke/TIA diagnoses in our sample underlines the importance for rapid and accurate triage of dizzy patients in the ED. Our approach (Figure 3) is highly sensitive (98.6\%), but of poor specificity (45.9\%). Only one stroke out of 32 verified by brain scan was missed, which outperforms misdiagnosis rates of $35 \%$ and more reported earlier (6-9). One reason is our conservative approach in unclear cases in the ED: until a peripheral cause is not proven, a central cause is assumed. Therefore, two-thirds of the cases were primarily admitted to stroke units (171/255 cases). Retrospectively, this was 


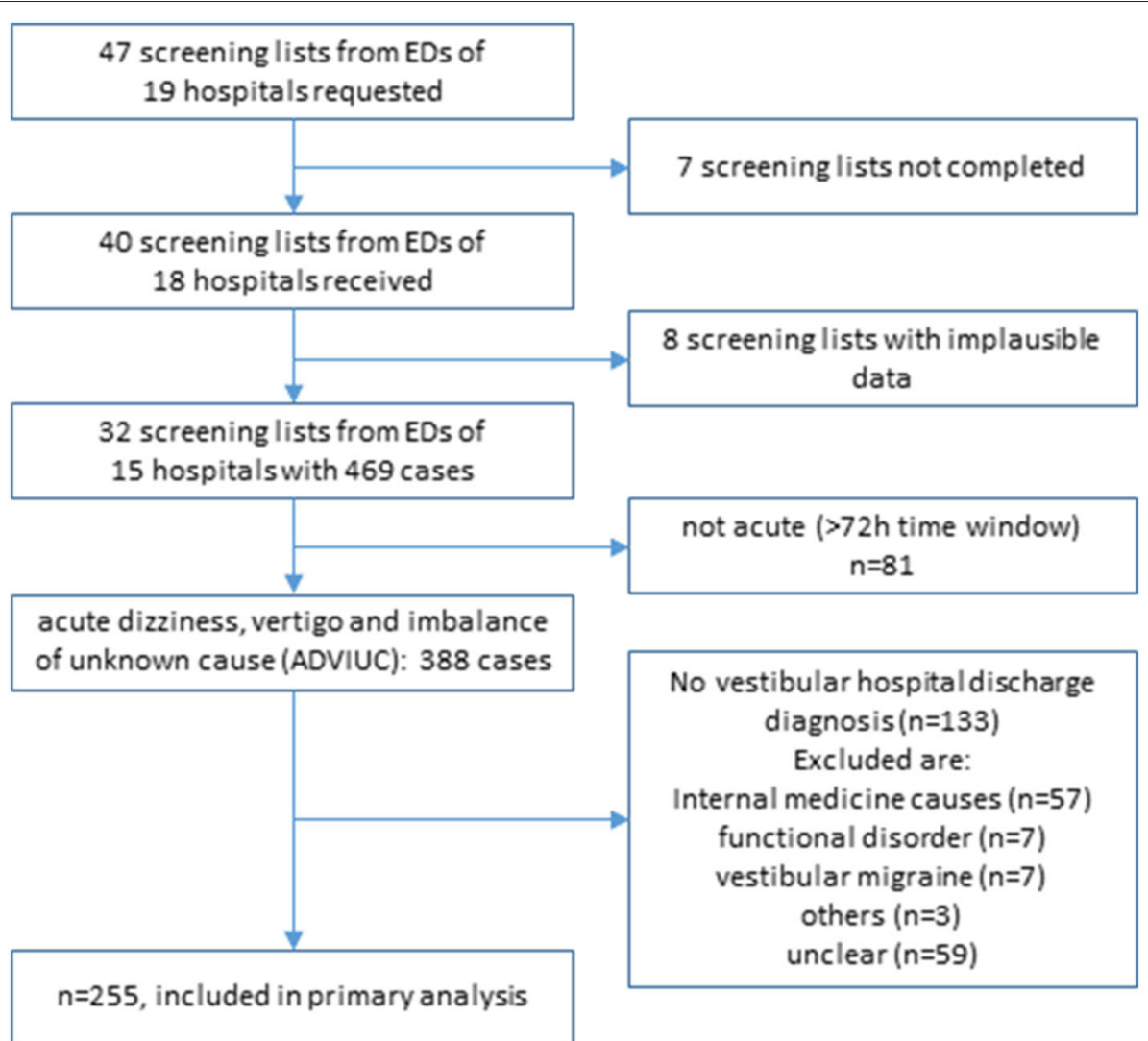

FIGURE 4 | Flowchart of the study cohort.

TABLE 4 | Accuracy of ED triage algorithm in vestibular cases.

\begin{tabular}{lccc}
\hline ED diagnosis final diagnosis & $\begin{array}{c}\text { Central } \\
\text { vestibular }\end{array}$ & $\begin{array}{c}\text { Peripheral } \\
\text { vestibular }\end{array}$ & Total \\
\hline Central or unclear & 73 & 98 & 171 \\
Peripheral & 1 & 83 & 84 \\
Total & 74 & 181 & 255 \\
Sensitivity: & $98.6 \%$ & & \\
Specificity: & $45.9 \%$ & & \\
Positive predictive value: & $42.7 \%$ & & \\
Negative predictive value: & $98.8 \%$ & &
\end{tabular}

ED diagnoses compared to final discharge diagnoses.

unnecessary in $57 \%$ of cases (98/171). Further improvement in triage tools is mandatory to reduce overadmission to stroke unit.

During planning and implementation of our TeleVertigo project, a strong feeling for the need of a specific concept for the management of dizzy patients in the ED of primary care hospitals evolved. Spectrum of diagnoses, training and experience of ED physicians and on-site neurologists, and diagnostic resources differ relevantly from other settings like at the general practitioner, in outpatient clinics, and in academic and specialized vertigo centers. Therefore, data and approaches cannot be transferred easily between these settings. In the literature, we could not find a commonly accepted definition for the relevant symptoms in our ED setting. There are various and substantially different definitions for acute vestibular syndrome (AVS) $(4,5,12,32)$, mostly requiring a persistence of symptoms over at least $24 \mathrm{~h}$ and a nystagmus. There is not an accepted definition for acute vertigo. Furthermore, vertigo and dizziness are different symptoms by definition (29), but cannot be distinguished by anamnesis in the ED in a relevant percentage of cases $(33,34)$. Therefore, we suggest a new definition for use in the ED setting, which focuses on the diagnostically challenging cases. These are all patients with acute dizziness, vertigo, or imbalance of unknown cause (ADVIUC). We defined "acute" as symptoms persisting $<72 \mathrm{~h}$. The reason for this is the consideration that for strokes with onset $>72 \mathrm{~h}$ stroke unit treatment usually is no longer indicated and therefore rapid triage to stroke unit or normal ward is unnecessary. "Unknown cause" means without an obvious reason for these symptoms detectable in the first medical check by ED physicians at admission (like an obvious focal neurological deficit, acute respiratory distress or relevant tachycardia). This excludes also recurrent manifestations of an already diagnosed episodic disorder, as long as the acute symptoms are preknown. In our cohort of ADVIUC cases in the ED, any nystagmus was detected in $51 \%$ of cases only. Cases without nystagmus $(n=189)$ correspond mostly to cases with dizziness of internal medicine cause (52/189), 

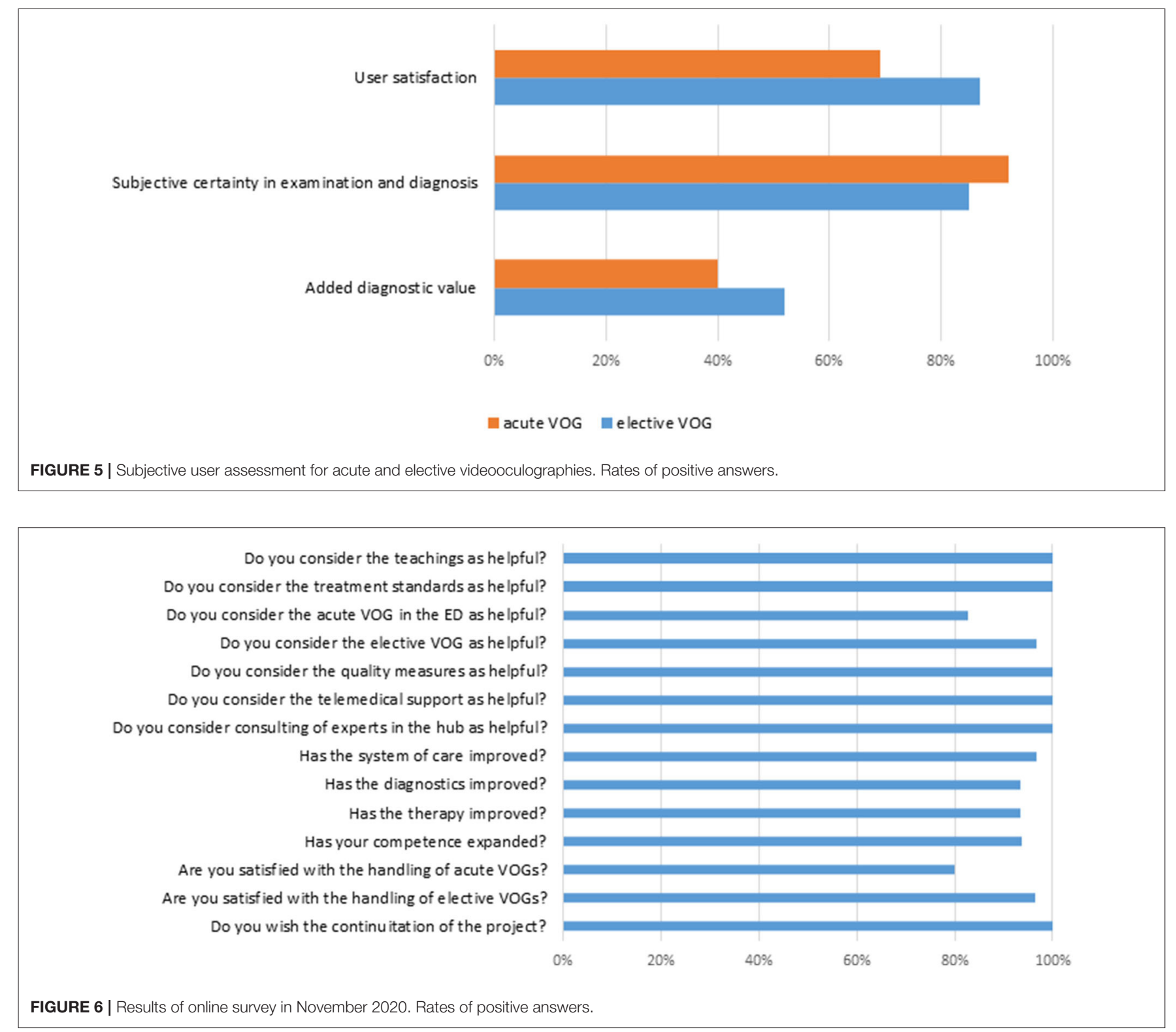

stroke/TIA (42/189), and unclear cases (44/189) and emphasize the difference between AVS and ADVIUC.

We believe that for these ADVIUC patients in this setting, a comprehensive approach for adequate diagnostic and therapy is required. The diagnostic approaches based on the HINTS rule alone do not seem sufficient and feasible. Diagnostic accuracy has only been shown in highly selected patients due to tight inclusion criteria (35). The HINTS rule does not work at all in cases with completely resolved symptoms such as TIA or BPPV. The generalizability of the positive study results to less selective cohorts appears problematic. Furthermore, in our experience, an independent application and interpretation of the HINTS rule by ED physicians in primary care hospitals rarely yields reliable results. Nevertheless, HINTS is a precious diagnostic tool and is implemented in our ED triage algorithm as well (see Figure 3). Essentially, it is embedded in a telemedically supported and network-based application to assure specific feasibility. Moreover, questions about internal medicine cause and Dix-Hallpike maneuvers are added, and the workflow is optimized. The teachings and trainings for teleconsultants, ED-physicians, on-site neurologists, technicians, and physiotherapists are focused on history-taking [especially about timing and triggers according to the TiTrATE concept $(36,37)]$, the examination of subtle focal neurological symptoms and central oculomotor signs and also the four major diagnoses involved (vertigo/dizziness of central cause, vestibular neuritis, BPPV and, internal medicine causes of dizziness). We assume that this knowledge is important to understand diagnostic and treatment standards and therefore improves adherence to standards and quality of care.

One strength of this study is the prospective data sampling with active screening of all ED cases. Consequently, we can report 
reliable numbers of cases of difficult dizziness presentations in the ED of primary care hospitals and also a reliable diagnostic spectrum. Hospital discharge diagnoses in the spokes were made thoroughly based on the established treatment standards and under supervision of the network center. Accuracy of the ED triage algorithm was prospectively analyzed.

Some limitations have to be addressed. First, although hospital discharge diagnoses in the spokes were made in the best way possible, the diagnostic quality does not reach standards of specialized academic centers and leaves room for misdiagnoses. MRI scans were only conducted on cases with suspicion for a central etiology; therefore, some central lesions may have been missed. Furthermore, analysis of accuracy of the ED triage was restricted to cases with a vestibular final discharge diagnosis, as the major challenge in the ED is the correct identification of stroke and TIA cases in ongoing but also resolved dizziness symptoms. Finally, we did not verify the proficiency of all staff members involved and did not systematically survey the quality of VOG examinations and diagnoses in the spoke hospitals. This system is aimed to improve quality of care in a large area with a clearly defined protocol to serve as many patients as possible. This expansion of competence into the area may be at the expense of the competence of the individual actors - a conflict you often face when setting up large scale systems of care. In diagnoses with potentially time dependent treatment (such as acute stroke), this strategy has been proven to be effective (18). The overall system, which includes the protocol and proficiency of all staff involved, seems very reassuring regarding the most important aim, the detection of an acute central cause.

In conclusion, the concept evaluated shows good acceptance for a complex telemedical and network-based approach to manage patients with acute dizziness, vertigo, or imbalance of unknown cause in the ED of primary care hospitals. Identification of stroke cases is accurate, whereas specificity needs further improvement. The concept could be a major step toward a broadly available evidence-based and state of the art diagnostics and therapy for patients with ADVIUC, especially in primary care hospitals of rural areas.

\section{DATA AVAILABILITY STATEMENT}

The raw data supporting the conclusions of this article will be made available by the authors, without undue reservation.

\section{REFERENCES}

1. Saber Tehrani AS, Coughlan D, Hsieh YH, Mantokoudis G, Korley FK, Kerber $\mathrm{KA}$, et al. Rising annual costs of dizziness presentations to US emergency departments. Acad Emerg Med. (2013) 20:689-96. doi: 10.1111/acem. 12168

2. Cnyrim CD, Newman-Toker D, Karch C, Brandt T, Strupp M. Bedside differentiation of vestibular neuritis from central "vestibular pseudoneuritis". J Neurol Neurosurg Psychiatry. (2008) 79:458-60. doi: 10.1136/jnnp.2007.123596

3. Eagles D, Stiell IG, Clement CM, Brehaut J, Kelly A-M, Mason S, et al. International survey of emergency physicians' priorities for clinical decision rules. Acad Emerg Med. (2008) 15:177-82. doi: 10.1111/j.1553-2712.2008. 00035.x

\section{ETHICS STATEMENT}

Ethical review and approval was not required for the study on human participants in accordance with the local legislation and institutional requirements. Written informed consent for participation was not required for this study in accordance with the national legislation and the institutional requirements.

\section{AUTHOR CONTRIBUTIONS}

PM-B, CL, NH, and GH contributed to conception and design of the study. CL and PM-B organized the database and performed the statistical analysis. PM-B wrote the first draft of the manuscript. All authors contributed to manuscript revision, read, and approved the submitted version.

\section{FUNDING}

NS-B, CL, JP, and PM-B efforts were supported by a grant from the Bavarian Ministry of Health and the German Foundation for Neurology (DSN).

\section{ACKNOWLEDGMENTS}

The authors thank all colleagues in the spoke hospitals and all teleconsultants involved in the TeleVertigo project. Participating centers were Munich Clinic Harlaching, InnKlinikum Altötting and the University of Regensburg. Participating spokes were Krankenhaus Agatharied, Kreisklinik Bad Reichenhall, Asklepios Stadtklinik Bad Tölz, Asklepios Klinik Burglengenfeld, Krankenhaus Cham, Donau-Isar-Klinikum Dingolfing, Kreisklinik Ebersberg, Krankenhaus Eggenfelden, Klinikum Landkreis Erding, Klinikum Freising, Krankenhaus Grafenau-Freyung, GoldbergKlinik Kelheim, InnKlinikum Mühldorf am Inn, RoMed Klinikum Rosenheim, Krankenhaus Rotthalmünster, Klinikum Traunstein, Krankenhaus Vilsbiburg, kbo-Inn-Salzach-Klinikum Wasserburg am Inn, Arberlandklinik Zwiesel. Authors also thank Dr. Tessa Marzotto Caotorta for her careful language editing of the manuscript.

4. Ljunggren M, Persson J, Salzer J. Dizziness and the acute vestibular syndrome at the emergency department: a population-based descriptive study. Eur Neurol. (2018) 79:5-12. doi: 10.1159/000481982

5. Newman-Toker DE, Hsieh Y-H, Camargo CA, Pelletier AJ, Butchy GT, Edlow JA. Spectrum of dizziness visits to US emergency departments: cross-sectional analysis from a nationally representative sample. Mayo Clin Proc. (2008) 83:765-75. doi: 10.4065/83.7.765

6. Kerber KA, Brown DL, Lisabeth LD, Smith MA, Morgenstern LB. Stroke among patients with dizziness, vertigo, and imbalance in the emergency department: a population-based study. Stroke. (2006) 37:24847. doi: 10.1161/01.STR.0000240329.48263.0d

7. Paul NL, Simoni M, Rothwell PM. Transient isolated brainstem symptoms preceding posterior circulation stroke: a population-based study. Lancet Neurol. (2013) 12:65-71. doi: 10.1016/S1474-4422(12)70299-5 
8. Moulin T, Sablot D, Vidry E, Belahsen F, Berger E, Lemounaud P, et al. Impact of emergency room neurologists on patient management and outcome. Eur Neurol. (2003) 50:207-14. doi: 10.1159/000073861

9. Tarnutzer AA, Lee S-H, Robinson KA, Wang Z, Edlow JA, NewmanToker DE, et al. misdiagnosis of cerebrovascular events in the era of modern neuroimaging: a meta-analysis. Neurology. (2017) 88:146877. doi: 10.1212/WNL.0000000000003814

10. Newman-Toker D, Curthoys I, Halmagyi G. Diagnosing stroke in acute vertigo: the HINTS family of eye movement tests and the future of the "Eye ECG”. Semin Neurol. (2015) 35:506-21. doi: 10.1055/s-0035-1564298

11. Newman-Toker DE, Kerber KA, Hsieh Y-H, Pula JH, Omron R, Saber Tehrani AS, et al. HINTS outperforms ABCD2 to screen for stroke in acute continuous vertigo and dizziness. Goldstein JN Acad Emerg Med. (2013) 20:986-96. doi: 10.1111/acem.12223

12. Kattah JC, Talkad AV, Wang DZ, Hsieh Y-H, Newman-Toker DE. HINTS to diagnose stroke in the acute vestibular syndrome: three-step bedside oculomotor examination more sensitive than early MRI diffusion-weighted imaging. Stroke. (2009) 40:3504-10. doi: 10.1161/STROKEAHA.109.55 1234

13. Kattah JC. Use of HINTS in the acute vestibular syndrome. An Overview. Stroke Vasc Neurol. (2018) 3:190-96. doi: 10.1136/svn-2018-000160

14. Carmona S, Martínez C, Zalazar G, Moro M, Batuecas-Caletrio A, Luis L, et al. The diagnostic accuracy of truncal ataxia and HINTS as cardinal signs for acute vestibular syndrome. Front Neurol. (2016) 7:125. doi: 10.3389/fneur.2016.00125

15. Mantokoudis G, Saber Tehrani AS, Wozniak A, Eibenberger K, Kattah JC, Guede CI, et al. VOR gain by head impulse videooculography differentiates acute vestibular neuritis from stroke. Otol Neurotol. (2015) 36:457-65. doi: 10.1097/MAO.00000000000 00638

16. Newman-Toker DE, Saber Tehrani AS, Mantokoudis G, Pula JH, Guede CI, Kerber KA, et al. Quantitative video-oculography to help diagnose stroke in acute vertigo and dizziness: toward an ECG for the eyes. Stroke. (2013) 44:1158-61. doi: 10.1161/STROKEAHA.111.000033

17. Celebisoy N. Acute vestibular syndrome: clinical head impulse test versus video head impulse test. J Neurol. (2018) 265:447. doi: 10.1007/s00415-018-8804-0

18. Audebert HJ, Schenkel J, Heuschmann PU, Bogdahn U, Haberl RL, Telemedic Pilot Project for Integrative Stroke Care Group. Effects of the implementation of a telemedical stroke network: the Telemedic Pilot Project for Integrative Stroke Care (TEMPiS) in Bavaria, Germany. Lancet Neurol. (2006) 5:7428. doi: 10.1016/S1474-4422(06)70527-0

19. Müller-Barna P, Hubert GJ, Boy S, Bogdahn U, Wiedmann S, Heuschmann PU, et al. Telestroke units serving as a model of care in rural areas: 10-year experience of the telemedical project for integrative stroke care. Stroke. (2014) 45:2739-44. doi: 10.1161/STROKEAHA.114.006141

20. Zee D, Newman-Toker D, Tourkevich R, Brune A, Green K, Peterson S, et al. Diagnostic impact of a device-enabled remote "Tele-Dizzy" consultation service. Neurology. (2020) 94 (15 Supplement).

21. Parker TM, Farrell N, Otero-Millan J, Kheradmand A, McClenney A, Newman-Toker DE. Proof of Concept for an "eyePhone" app to measure video head impulses. Digit Biomark. (2020) 5:1-8. doi: 10.1159/000511287

22. Acute Video-oculography for Vertigo in Emergency Rooms for Rapid Triage (AVERT). ClinicalTrials.gov (2021). Available online at: https://clinicaltrials. gov/ct2/show/record/NCT02483429 (accessed July 23, 2021).

23. Müller-Barna $P$, Hubert ND, Bergner C, Schütt-Becker N, Rambold $\mathrm{H}$, Haberl RL, et al. Televertigo: diagnosing stroke in acute dizziness: a telemedicine-supported approach. Stroke. (2019) 50:3293-8. doi: 10.1161/STROKEAHA.119.026505

24. von Martial R, Leinweber C, Hubert N, Rambold H, Haberl RL, Hubert GJ, et al. Feasibility of telemedical HINTS (head impulse-nystagmus-test of skew) evaluation in patients with acute dizziness or vertigo in the emergency department of primary care hospitals. Front Neurol. (2021) 12:768460. doi: 10.3389/fneur.2021.768460

25. DESTATIS - Federal Statistical Office. (2019). Available online at: https://www.destatis.de/DE/Themen/Laender-Regionen/Regionales/ Gemeindeverzeichnis/Administrativ/04-kreise.html (accessed June 2021).
26. Govindarajan R, Anderson ER, Hesselbrock RR, Madhavan R, Moo LR, Mowzoon N, et al. Developing an outline for teleneurology curriculum: AAN telemedicine work group recommendations. Neurology. (2017) 89:9519. doi: 10.1212/WNL.0000000000004285

27. Eggers SDZ, Bisdorff A, von Brevern M, Zee DS, Kim J-S, Perez-Fernandez N, et al. Classification of vestibular signs and examination techniques: nystagmus and nystagmus-like movements: consensus document of the committee for the international classification of vestibular disorders of the bárány society. $J$ Vestib Res. (2019) 29:57-87. doi: 10.3233/VES-190658

28. von Brevern M, Bertholon P, Brandt T, Fife T, Imai T, Nuti D, et al. Benign paroxysmal positional vertigo: diagnostic criteria: consensus document of the committee for the classification of vestibular disorders of the bárány society. $J$ Vestib Res. (2015) 25:105-17. doi: 10.3233/VES-150553

29. Bisdorff A, Von Brevern M, Lempert T, Newman-Toker DE. Classification of vestibular symptoms: towards an international classification of vestibular disorders. J Vestib Res. (2009) 19:1-13. doi: 10.3233/VES-2009-0343

30. Hubert GJ, Meretoja A, Audebert HJ, Tatlisumak T, Zeman F, Boy S, et al. Stroke thrombolysis in a centralized and a decentralized system (helsinki and telemedical project for integrative stroke care network). Stroke. (2016) 47:2999-3004. doi: 10.1161/STROKEAHA.116.014258

31. Zwergal A, Dieterich M. Vertigo and dizziness in the emergency room. Curr Opin Neurol. (2020) 33:117-25. doi: 10.1097/WCO.0000000000000769

32. Venhovens J, Meulstee J, Verhagen WIM. Acute vestibular syndrome: a critical review and diagnostic algorithm concerning the clinical differentiation of peripheral versus central aetiologies in the emergency department. J Neurol. (2016) 263:2151-7. doi: 10.1007/s00415-016-8081-8

33. Newman-Toker DE, Cannon LM, Stofferahn ME, Rothman RE, Hsieh Y-H, Zee DS. Imprecision in patient reports of dizziness symptom quality: a crosssectional study conducted in an acute care setting. Mayo Clin Proc. (2007) 82:1329-40. doi: 10.4065/82.11.1329

34. Tarnutzer AA, Berkowitz AL, Robinson KA, Hsieh Y-H, Newman-Toker DE. Does my dizzy patient have a stroke? a systematic review of bedside diagnosis in acute vestibular syndrome. Can Med Assoc J. (2011) 183:E57192. doi: $10.1503 / \mathrm{cmaj} .100174$

35. Ohle R, Montpellier R, Marchadier V, Wharton A, McIsaac S, Anderson $\mathrm{M}$, et al. Can emergency physicians accurately rule out a central cause of vertigo using the HINTS examination? a systematic review and meta-analysis. Herausgeber Acad Emerg Med. (2020) 27:887-96. doi: 10.1111/acem.13960

36. Newman-Toker DE, Edlow JA. TiTrATE. Neurol Clin. (2015) 33:57799. doi: 10.1016/j.ncl.2015.04.011

37. Edlow JA, Gurley KL, Newman-Toker DE. A new diagnostic approach to the adult patient with acute dizziness. J Emerg Med. (2018) 54:46983. doi: 10.1016/j.jemermed.2017.12.024

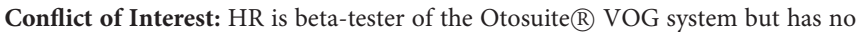
financial interest in the product. He received honorary from Natus Medical ApS, Denmark, and Henning-Arzneimittel, Germany. PM-B, CL, JP, and NS-B report grants from the Bavarian Ministry of Health and the German Foundation for Neurology DSN during the conduct of the study.

The remaining authors declare that the research was conducted in the absence of any commercial or financial relationships that could be construed as a potential conflict of interest.

Publisher's Note: All claims expressed in this article are solely those of the authors and do not necessarily represent those of their affiliated organizations, or those of the publisher, the editors and the reviewers. Any product that may be evaluated in this article, or claim that may be made by its manufacturer, is not guaranteed or endorsed by the publisher.

Copyright (C) 2022 Müller-Barna, Leinweber, Pfaffenrath, Schütt-Becker, von Martial, Greck, Hubert, Rambold, Haberl and Hubert. This is an open-access article distributed under the terms of the Creative Commons Attribution License (CC BY). The use, distribution or reproduction in other forums is permitted, provided the original author(s) and the copyright owner(s) are credited and that the original publication in this journal is cited, in accordance with accepted academic practice. No use, distribution or reproduction is permitted which does not comply with these terms. 